\title{
Organized Current Patterns in Disordered Conductors
}

\author{
Y. Japha ${ }^{1}$, O. Entin-Wohlman ${ }^{1}$, T. David ${ }^{1}$, R. Salem ${ }^{1}$, \\ S. Aigner ${ }^{2,3}$, J. Schmiedmayer ${ }^{2,3}$, and R. Folman ${ }^{1}$ \\ ${ }^{1}$ Department of Physics, Ben-Gurion University of the Negev, P.O. Box 653, Be'er-Sheva 84105, Israel. \\ ${ }^{2}$ Physikalisches Institut, Universität Heidelberg, Philosophenweg 12, 69120 Heidelberg. \\ ${ }^{3}$ Atominstitut der sterreichischen Universitten, TU-Wien, Stadionalle 2, 1020 Vienna, Austria. \\ (Dated: November 1, 2018)
}

\begin{abstract}
We present a general theory of current deviations in straight current carrying wires with random imperfections, which quantitatively explains the recent observations of organized patterns of magnetic field corrugations above micron-scale evaporated wires. These patterns originate from the most efficient electron scattering by Fourier components of the wire imperfections with wavefronts along the $\pm 45^{\circ}$ direction. We show that long range effects of surface or bulk corrugations are suppressed for narrow wires or wires having an electrically anisotropic resistivity.
\end{abstract}

PACS numbers: 73.50.Bk,03.75.Be,37.10.Gh,05.40.-a

Electron scattering by microscopic structural imperfections in thin conducting films is a major factor determining their conductivity properties, especially at low temperatures.2. Orginary polycrystalline metal wires with straight boundaries are usually considered to have ohmic conductance with a homogeneous current flow on a scale much larger than their grain size (typically tens of nanometers $)^{3}$. Measurements using ultracold atoms as a highly sensitive probe to minute changes in the magnetic field have revealed directional deviations of the current flow far from the edges of the wire ${ }^{4}$. Recent observations of atomic density fluctuations a few microns above wires of different thickness and grain size have revealed organized patterns of current flow directional deviations which are oriented predominantly at $\pm 45^{\circ}$ relative to the wire axis, on a length scale as large as tens of microns ${ }^{5}$. It was shown that this effect is a general property of electron scattering by random imperfections in the conductor. In contrast to previous observations of atomic density fluctuations above current carrying wires $6,7,8,9,10$, which were attributed to current irregularities due to wire edge corrugations $10.11,12$, the recent observations emphasize the importance of the wire surface or bulk structural imperfections on a length scale of the order of a micron or longer.

Here, we present a detailed model for the current irregularities formed in a current carrying wire with random geometrical perturbations or bulk resistivity inhomogeneities. This model enables not only the quantitative understanding of the observed patterns and their origin, but also provides predictions of electron transport properties in wires with various geometries and crystalline structures. Together with further measurements using the ultracold atomic probe, it is expected to shed new light on electron transport, and to allow for less corrugated atomic traps and guides to be developed for atom optics and quantum technology $\underline{13}$.

The results of our calculations are demonstrated in Fig. 1 comparing the measured atomic density patterns with patterns calculated by assuming random imperfec-

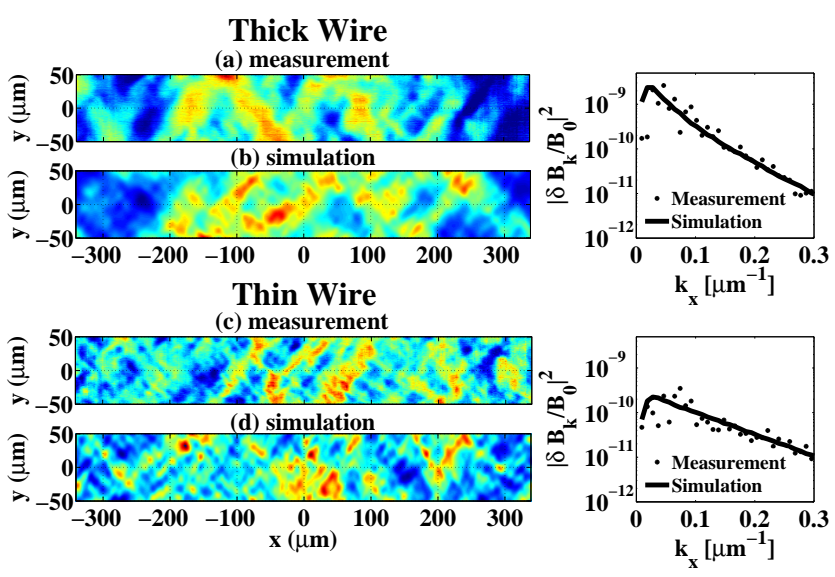

FIG. 1: Measured 5 and simulated patterns of atomic density (normalized) $\sim 3.5 \mu \mathrm{m}$ above $(\mathrm{a}-\mathrm{b})$ a thick wire $(H=2 \mu \mathrm{m})$, and $(\mathrm{c}-\mathrm{d})$ a thin wire $(H=280 \mathrm{~nm})$, with grain size of $\sim 70 \mathrm{~nm}$ and $\sim 40 \mathrm{~nm}$, respectively. Atomic density variations measure corrugations in the magnetic field component $\delta B_{x}$ along the wire axis $\hat{x}$. These corrugations are due to current directional deviations from the main current along $\hat{x}$. The trapping potential ensures a cloud width of $\sim 1 \mu \mathrm{m}$ along $\hat{y}$ and $\hat{z}$, and hundreds of $\mu \mathrm{m}$ in the $\hat{x}$ direction. To create the above $2 \mathrm{~d}$ maps, the different transverse locations are scanned across the wire at a constant height. The simulation assumes a combination of bulk resistivity inhomogeneity and geometrical perturbations of the wire with parameters chosen such that the power spectrum of the magnetic field along $\hat{x}$, averaged over $\hat{y}$ and over many realizations of the simulation, fits the measured power spectrum (right).

tions of the wire geometry or bulk resistivity. Each spectral (Fourier) component of these imprefections is a plane wave with a random phase and an amplitude taken from a non-white isotropic power spectrum modeled in Ref. 5 . The angular preference of the patterns emerges from a universal electron scattering mechanism described below, where the apparent difference in the spectral composition of the density fluctuations above the thick wire of 
(a)

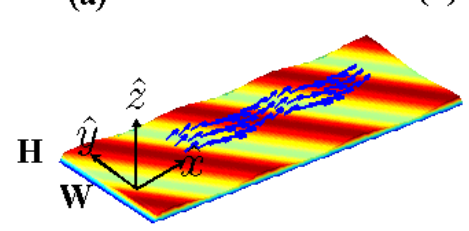

(c)

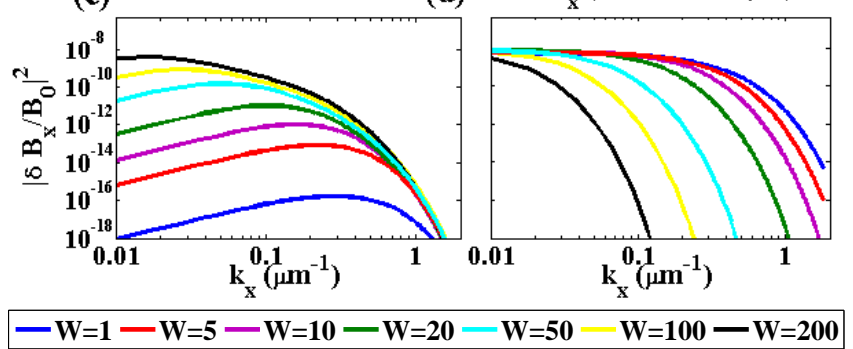

FIG. 2: (a) A single Fourier component (plane wave) of the planar resistivity perturbations due to bulk inhomogeneity or wire thickness variations induces current flow directional changes (arrows). The current tilts along low resistivity wavefronts and across high resistivity wavefronts. (b) The amplitudes of transverse current component $\delta J_{y}(\boldsymbol{\kappa})\left[\boldsymbol{\kappa} \equiv\left(k_{x}, k_{y}\right)\right]$, generated by resistivity perturbations $\delta \rho(\kappa) \propto 1 / \kappa$ (see Ref. 5), are proportional to $\sin 2 \theta \kappa$ (color scheme). For a wire with a finite length $L$ and width $W$, these amplitudes are calculated at discrete values of $\kappa$, which are integer products of $2 \pi / L$ and $2 \pi / W$. (c) As a consequence of this discreteness, the corrugations at long wavelengths $\left(k_{x} W<1\right)$ are suppressed when the wire becomes narrower: the density of $k_{y}$ states becomes lower and no corresponding values of $k_{y}$ exist along the maximum scattering amplitude line $k_{y} \sim k_{x}$. Here, the power spectrum of the magnetic field corrugations at $3.5 \mu \mathrm{m}$ above the center of the wire is shown as a function of $k_{x}$ for resistivity perturbations $\delta \rho_{\boldsymbol{\kappa}} / \rho_{0}=3.4 \cdot 10^{-4}\left(\kappa_{0} / \kappa\right)$ with $\kappa_{0}=2 \pi / 680 \mu \mathrm{m}^{-1}$. (d) For comparison, the magnetic field corrugations above the center of the wire are shown for wires of different widths for a model assuming edge fluctuations with $\left.\left|\delta y_{ \pm}\left(k_{x}\right)\right|=10 \mathrm{~nm} \times\left(\kappa_{0} / k_{x}\right)\right]$. Here, short wavelength components are suppressed when the wire becomes wider.

thickness $2 \mu \mathrm{m}$ (Fig. 1 $\mathrm{h}-\mathrm{b}$ ) and the thin wire of thickness 280nm (Fig. 1 1 -d) is attributed to the different nature of the wire imperfections.

Our calculations are performed for a metallic wire as in Fig. 2 2 , having a rectangular cross-section of width $W$ in the $\hat{\mathbf{y}}$ direction and thickness $H$ in the $\hat{\mathbf{z}}$ direction, and carrying a current density $\mathbf{J}(\mathbf{r})=J_{0} \hat{\mathbf{x}}+\delta \mathbf{J}(\mathbf{r})$. Here $J_{0}=I / W H$ is the regular current density for a total current $I$. Utilizing Ohm's law $\mathbf{J}=\mathbf{E} / \rho$ where $\mathbf{E}$ is the electric field, and Maxwell's equation $\boldsymbol{\nabla} \times \mathbf{E}=0$, we find

$$
\nabla \times \mathbf{J}=-\frac{\nabla \rho}{\rho} \times \mathbf{J}
$$

with $\rho=\rho_{0}+\delta \rho(\mathbf{r})$ being the isotropic resistivity. Although significant resistivity perturbations may exist in a polycrystalline metal near grain boundaries (length scale of nanometers), one may safely assume that over most of the length scales of interest (microns) $\delta \rho \ll \rho$, such that in the Fourier expansion $\delta \rho(\mathbf{r})=\sum_{\mathbf{k}} \rho_{\mathbf{k}} e^{i \mathbf{k} \cdot \mathbf{r}}$ one has $\left|\rho_{\mathbf{k}}\right| \ll \rho_{0}$ for any relevant wave number $\mathbf{k} \equiv\left(k_{x}, k_{y}, k_{z}\right)$. By keeping only terms up to first order in the resistivity gradient $\nabla \rho$ and using the current continuity equation $\boldsymbol{\nabla} \cdot \delta \mathbf{J}=0$ we obtain the solution for the components of the current irregularities as a function of the bulk inhomogeneity

$$
\delta \mathbf{J}^{(\text {bulk })}(\mathbf{k})=J_{0}\left(\frac{k_{x}}{|\mathbf{k}|^{2}} \mathbf{k}-\hat{\mathbf{x}}\right) \frac{\delta \rho_{\mathbf{k}}}{\rho_{0}},
$$

where the transverse components $\left(k_{y}, k_{z}\right)$ of the wave vector $\mathbf{k}$ take the discrete values $2 \pi(m / W, n / H)$ with integers $m$ and $n,-\infty<m, n<\infty$.

The horizontal transverse current irregularities $\delta J_{y}^{\text {(bulk) }}$ are proportional to $k_{x} k_{y} / k^{2} \propto \sin 2 \theta_{\mathbf{k}}$, where $\theta_{\mathbf{k}} \equiv$ $\tan ^{-1}\left(k_{y} / k_{x}\right)$ is the angle in the $\mathrm{x}-\mathrm{y}$ plane. This immediately implies that transverse currents are predominantly generated by Fourier components of the resistivity perturbations with wavefronts oriented at $\pm 45^{\circ}$. Vertical current irregularities $\delta J_{z}^{\text {(bulk) }}$ are proportional to $k_{x} k_{z} / k^{2} \propto \sin 2 \phi_{\mathbf{k}}$, where $\phi_{\mathbf{k}} \equiv \tan ^{-1}\left(k_{z} / k_{x}\right)$. Vertical currents are therefore significant only for Fourier components satisfying $k_{x} \sim k_{z}\left(\phi_{\mathbf{k}} \sim \pm 45^{\circ}\right)$, namely, for longitudinal wavelengths $2 \pi / k_{x}$ of the order of the thickness $H$ or less, corresponding to non-zero values of $k_{z}$. For thin wires, these wavelengths are usually beyond the spatial measurement resolution in the $x-y$ plane. At wavelengths of interest, much larger than $H\left(k_{x} \ll k_{z}\right)$, vertical currents are suppressed as $\delta J_{z}^{(\text {bulk })} \propto \frac{k_{x} H}{2 \pi} \ll 1$.

In the following we refer to the spectral regime $\left(k_{x}, k_{y}\right) \ll 2 \pi / H$ as the "thin film limit", where only contributions from Fourier terms with $k_{z}=0$ are important. We will then consider the film as two-dimensional and characterize it by the real-space vector $\boldsymbol{\xi} \equiv(x, y)$ and Fourier space vector $\boldsymbol{\kappa} \equiv\left(k_{x}, k_{y}\right)$. Thickness variations of the wire $\delta H(x, y)$ may then be regarded as irregularities of the thin film resistivity $\delta \rho^{\text {thickness }}=-\rho_{0} \delta H / H$. Figure 2 2 demonstrates the generation of periodic horizontal current directional deviations due to resistivity perturbations originating from bulk or thickness variations.

A typical magnetic potential along an elongated trap, such as that used in Ref. 5, is determined mainly by the longitudinal component of the magnetic field fluctuations at the trapping position. Its Fourier spectrum at a height $z_{0}$ is related to the current irregularities in the wire by

$$
\begin{aligned}
& \delta B_{x}\left(k_{x}, k_{y}, z_{0}\right)=\frac{\mu_{0}}{2} \int_{-H}^{0} d z^{\prime} e^{-\kappa\left|z_{0}-z^{\prime}\right|} \times \\
& \times\left[\delta J_{y}\left(k_{x}, k_{y}, z^{\prime}\right)+i \sin \theta \kappa \delta J_{z}\left(k_{x}, k_{y}, z^{\prime}\right)\right],
\end{aligned}
$$

where $\mu_{0}$ is the permeability of the vacuum. Here, the free space (continuous) Fourier transformations $\delta \mathbf{J}\left(k_{x}, k_{y}, z^{\prime}\right)$ may be approximated by their discrete form as in Eq. (22) if $\kappa W \gg 1$ and $z_{0} \ll W / 2$. Substituting $\delta \mathbf{J}$ of Eq. (2) into this expression one finds that $\delta B_{x}(\boldsymbol{\kappa}) \propto e^{-\kappa z_{0}} \sin 2 \theta_{\boldsymbol{\kappa}}$ limiting the spatial resolution in the $\mathrm{x}-\mathrm{y}$ plane by the measurement distance $z_{0}$. 
The $\sin 2 \theta_{\kappa}$ dependence together with a $\sim 1 / \kappa$ dependence of the resistivity perturbations (found in the spectral analysis of the data in Ref. 5), demonstrated by the color map in Fig. 2 $\mathrm{b}$, describe well the behavior in the continuum limit $W \rightarrow \infty$. However, for finite widths (and a finite measurement length $L$ ) $k_{x}$ and $k_{y}$ assume only discrete values which are integer multiples of $2 \pi / L$ and $2 \pi / W$, respectively, as demonstrated by the grid of dots superposed on the color map. It follows that for small values of $k_{x}$, no counterparts $k_{y}$ exist on the grid which lie in the region where $\left|\sin 2 \theta_{\boldsymbol{\kappa}}\right|$ is large, or more specifically, around the line $\theta_{\kappa}=45^{\circ}$. This implies that at wavelengths larger than the wire width $W$ the current irregularities are significantly suppressed beyond the suppression caused by the reduction of grid points. This prediction is demonstrated in the power spectrum shown in Fig. 22. This result is very different from the effect of current irregularities due to edge roughness, which characterized measurements of atomic density fluctuations in some previous works $10,11,12$. In that case, the short wavelengths are exponentially suppressed near the center of the wire, while only wavelengths of the order of the wire width or more are effective (Fig. 2 $2 \mathrm{~d}$ ).

Another prediction of our model is obtained when we generalize the situation to the case where the conducting wire is electrically anisotropic, such that the resistivity is a diagonal tensor and Ohm's law generalizes to $E_{j}=\rho_{j} J_{j}$ for $j=x, y, z$. In this case Eq. (2) becomes 14

$$
\delta \mathbf{J}^{(\text {bulk })}(\mathbf{k})=J_{0}\left(\frac{k_{x}}{\mathbf{k} \cdot \mathbf{q}} \mathbf{q}-\hat{\mathbf{x}}\right) \frac{\delta \rho_{x, \mathbf{k}}}{\rho_{x, 0}},
$$

where $\mathbf{q}=\left(k_{x} / \rho_{x}, k_{y} / \rho_{y}, k_{z} / \rho_{z}\right)$. In the limit of a thin film, where $k_{z}=0$, the horizontal transverse current irregularities $\delta J_{y}$ are proportional to $\sin 2 \theta_{\boldsymbol{\kappa}} /(1+(r-$ 1) $\cos ^{2} \theta_{\boldsymbol{\kappa}}$ ), where $r=\rho_{y} / \rho_{x}$ is the resistivity ratio. As demonstrated in Fig. 3, the scattering at angles $\theta_{\boldsymbol{\kappa}}<45^{\circ}$ is suppressed if $r>1$ and enhanced if $r<1$, thus changing the preferred scattering wavefront angle in the range $0^{\circ}<\theta_{\boldsymbol{\kappa}}<90^{\circ}$. The overall magnetic corrugations are suppressed as $r^{-3 / 4}$ in the limit of high anisotropy $r \gg 1$.

Now we turn to a more detailed theory of current irregularities due to geometrical imperfections of the wire ${ }^{15}$. We solve Eq. (10) with $\delta \rho \rightarrow 0$ and with boundary conditions ensuring that the current flows parallel to the boundaries. Taking the upper and lower surfaces of the wire at $z= \pm H / 2+\delta z_{ \pm}$and the right and left edges at $y= \pm W / 2+\delta y_{ \pm}$, where $\delta z_{ \pm}(x, y)$ and $\delta y_{ \pm}(x, z)$ are small fluctuations of the corresponding surfaces, we obtain the following boundary conditions,

$$
\begin{aligned}
& \delta J_{y}\left(x, \pm \frac{W}{2}, z\right)=J_{0} \frac{\partial \delta y_{ \pm}}{\partial x}, \\
& \delta J_{z}\left(x, y, \pm \frac{H}{2}\right)=J_{0} \frac{\partial \delta z_{ \pm}}{\partial x},
\end{aligned}
$$

where terms of second or higher orders in $\delta z_{ \pm}$and $\delta y_{ \pm}$ were omitted. The current irregularities are then written

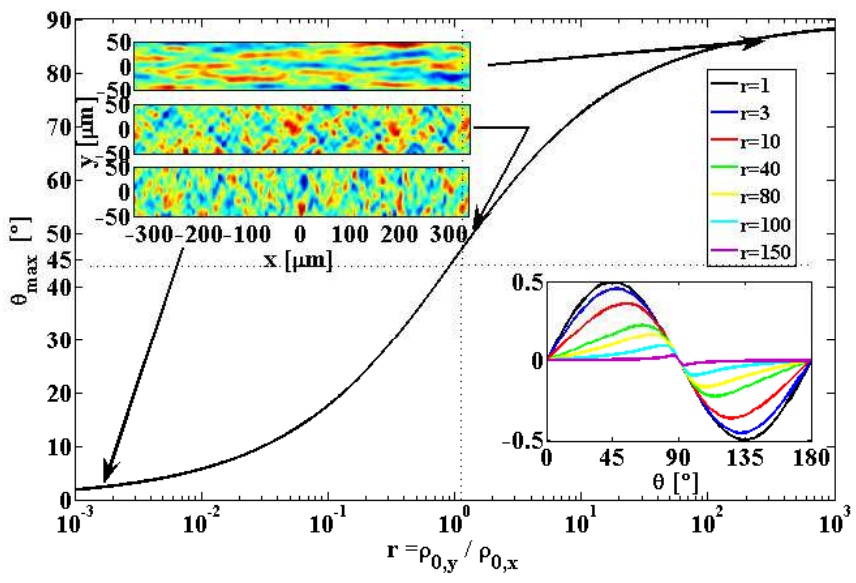

FIG. 3: For a current flowing through an electrically anisotropic wire, the perturbation wavefront angle $\theta_{\boldsymbol{\kappa}}^{\max }$, giving rise to maximum transverse electron scattering, will depend on the ratio $r=\rho_{y} / \rho_{x}$ between the transverse and longitudinal resistivities (main plot). The two-dimensional maps show the predicted atomic density above a wire similar to that presented in Fig. 1 in the extreme cases $\theta_{\kappa}=0^{\circ}(r \ll 1), 90^{\circ}$ $(r \gg 1)$ and the isotropic case $\theta \kappa=45^{\circ}(r=1)$. Bottom inset - the magnetic corrugation amplitude as a function of $\theta_{\boldsymbol{\kappa}}$, which is suppressed when $r>1$. For details see Ref. 14 .

as a sum of two terms,

$$
\delta \mathbf{J}^{(\operatorname{surf})}(\mathbf{r})=\sum_{k_{x}} e^{i k_{x} x}\left[\delta \mathbf{J}_{k_{x}}^{W}(y, z)+\delta \mathbf{J}_{k_{x}}^{H}(y, z)\right] .
$$

Eq. (11) with $\nabla \rho=0$ together with the continuity equation $\nabla \cdot \mathbf{J}=0$ imply that the current can be written as the gradient of a potential function $\delta \mathbf{J}^{\text {(surf) }}=\nabla \mathcal{F}$, which satisfies the Laplace equation $\nabla^{2} \mathcal{F}=0$. It follows that the terms in Eq. (6) have the form

$$
\begin{gathered}
\delta \mathbf{J}_{k_{x}}^{W}(y, z)=i k_{x} J_{0} \sum_{n, \pm} \mathbf{a}_{n, \pm}\left(k_{x}\right) e^{i 2 \pi n z / H} e^{-|y \mp W / 2| / \lambda_{n}}(7) \\
\delta \mathbf{J}_{k_{x}}^{H}(y, z)=i k_{x} J_{0} \sum_{m, \pm} \mathbf{b}_{m, \pm}\left(k_{x}\right) e^{i 2 \pi m y / W} e^{-|z \mp H / 2| / \lambda_{m}},(8)
\end{gathered}
$$

where the exponential terms describe the attenuation of current fluctuations induced by each boundary perturbation at a distance $\lambda_{n}=\left[k_{x}^{2}+(2 \pi n / H)^{2}\right]^{-1 / 2}$ from the left/right boundaries and $\lambda_{m}=\left[k_{x}^{2}+(2 \pi m / W)^{2}\right]^{-1 / 2}$ from the top/bottom boundaries. Since $\delta \mathbf{J}$ is derivable from a scalar function, it follows that each of the vectorial coefficients $\mathbf{a}_{n, \pm}$ and $\mathbf{b}_{m, \pm}$ can be derived from the corresponding scalar coefficients. Linear equations are obtained for these scalar coefficients when $\delta \mathbf{J}$ of Eq. (6) is substituted in the boundary conditions (5 $\mathbb{5}^{16}$.

Next, we describe the solutions of these equations for a few typical simple cases. The term $\delta \mathbf{J}_{k_{x}}^{W}$ (Eq. (7)) is significant when the edge perturbations $\delta y_{ \pm}$are large and the measurement height is comparable to the wire width $W$. This situation was discussed in previous works 11,12 . Here we concentrate on the other limit, where the field is 
measured at a low height and a large distance from the edges compared to $\lambda_{n}$ such that $\delta \mathbf{J}_{k_{x}}^{W} \sim 0$ for most values of $k_{x}$. The surface height fluctuations $\delta z_{\boldsymbol{\kappa}}^{ \pm}$then generate the following current irregularities (Eq. (8))

$$
\begin{aligned}
& \delta J_{y}^{(\operatorname{surf})}(\mathbf{r}) \approx-J_{0} \sum_{\boldsymbol{\kappa}} e^{i \boldsymbol{\kappa} \cdot \boldsymbol{\xi}} k_{x} \frac{k_{y}}{\kappa} \frac{\cosh (\kappa z)}{\sinh (\kappa H / 2)} \frac{\delta H_{\boldsymbol{\kappa}}}{2} \\
& \delta J_{z}^{(\text {surf })}(\mathbf{r}) \approx J_{0} \sum_{\boldsymbol{\kappa}} e^{i \boldsymbol{\kappa} \cdot \boldsymbol{\xi}_{i k_{x}}} \frac{\cosh (\kappa z)}{\cosh (\kappa H / 2)} \delta z_{\boldsymbol{\kappa}}^{\text {mean }}
\end{aligned}
$$

where $\delta H_{\boldsymbol{\kappa}}=\delta z_{\boldsymbol{\kappa}}^{+}-\delta z_{\bar{\kappa}}^{-}$are wire thickness variations and $\delta z_{\boldsymbol{\kappa}}^{\text {mean }}=\left(\delta z_{\boldsymbol{\kappa}}^{+}+\delta z_{\boldsymbol{\kappa}}^{-}\right) / 2$ are height fluctuations of the center of the wire. In the thin film limit $\kappa H \ll 1$, we find $\kappa z \ll 1$ for $|z| \leq H / 2$ such that $\delta J_{y}$ assumes a form similar to Eq. (2) with $\delta \rho_{\boldsymbol{\kappa}} / \rho_{0} \rightarrow-\delta H_{\boldsymbol{\kappa}} / H$

$$
\delta J_{y}^{(\operatorname{surf})}(\mathbf{r}) \approx-J_{0} \sum_{\boldsymbol{\kappa}} e^{i \boldsymbol{\kappa} \cdot \boldsymbol{\xi}} \sin 2 \theta \boldsymbol{\kappa} \frac{\delta H_{\boldsymbol{\kappa}}}{2 H} .
$$

In the same limit, the magnitude of the vertical current component $\delta J_{z}$ becomes negligible, since $\delta J_{z}^{\text {(surf) }} \propto$ $k_{x} \delta z_{\boldsymbol{\kappa}}^{\text {mean }}=k_{x} H\left(\delta z_{\boldsymbol{\kappa}}^{\text {mean }} / H\right) \ll \delta z_{\boldsymbol{\kappa}}^{\text {mean }} / H$.

Equation (11) shows that the surface roughness $\delta z_{ \pm}$ causes current irregularities mainly through the thickness variations $\delta H=\delta z_{+}-\delta z_{-}$. In thin films, long scale surface height variations $\delta z_{+}$, which follow bottom (wafer) surface variations $\delta z_{-}$, are not expected to cause significant current variations. On the other hand, the effect of thickness variations is expected to be more pronounced in thin films, where $\delta H / H$ is larger than in thicker wires. The power spectrum of the measured magnetic field pattern above the thin wire (Fig. 15), which was analyzed in Ref. 5, could be explained by a model assuming that thickness variations exist mainly at short length scales (below $\sim 20 \mu \mathrm{m}$ ), while $\delta z_{+} \approx \delta z_{-}$at long length scales. This may explain the shorter characteristic length scale of the features in the thin wire (Fig. 11) relative to the thick wire (Fig. 11), where the measured surface roughness was not sufficiently large to account for the magnetic field fluctuations even if the bottom surface variations were assumed to be uncorrelated with the top surface. This analysis implies that bulk resistivity perturbations with $\propto 1 / k$ spectrum could play an important role in the thick wire, but they are much smaller in the thin wire.

To conclude, we have presented a detailed model for current directional deviations in thin wires with random imperfections on a length scale of the order of a micron or longer. These deviations may arise either from bulk resistivity inhomogeneities or from geometrical perturbations of the wire, where the significance of each factor depends on the wire thickness and fabrication process. In both cases, electron scattering is dominant at wavefronts oriented at $\pm 45^{\circ}$ relative to the main current axis. The model predicts a strong suppression of long wavelength current deviations originating from bulk or surface corrugations in narrower wires. Electrically anisotropic materials are also capable of significantly suppressing these deviations. Such analysis opens the road for material engineering to considerably improve atom optics on atomchips where currents are used for creating magnetic potentials for atom trapping and guiding. Comparison of this theory with further cold atom magnetometry or other measurements providing high field sensitivities and spatial resolution, will enable deeper understanding of electron transport in thin films.

We thank the team of the Ben-Gurion University Weiss Family Laboratory for Nanoscale Systems (www.bgu.ac.il/nanofabrication) for the fabrication of the chip and Jürgen Jopp of the Ben-Gurion University Ilse Katz Center for Nanoscale Science for assisting with surface measurements. R.F. thanks Yoseph (Joe) Imry. We acknowledge support by the FWF, the DFG, the German Ministry of Education and Research (DIP), the EC 'atomchip' (RTN) consortium, the American-Israeli Foundation (BSF) and the Israeli Science Foundation.
1 J. M. Ziman, Electrons and Phonons, Oxford: Oxford University Press, 1960.

2 A. F. Mayadas and M. Shatzkes, Phys. Rev. B 1, 1382 (1970).

3 For a review of transport phenomena in random media see M. B. Isichenko, Rev. Mod. Phys. 64, 961 (1992).

${ }^{4}$ S. Wildermuth, S. Hofferberth, I. Lesanovsky, E. Haller, M. Andersson, S. Groth, I. Bar-Joseph, P. Krüger, and J. Schmiedmayer, Nature 435, 440 (2005).

5 S. Aigner, L. Della Pietra,Y. Japha, O. Entin-Wohlman, T. David, R. Salem, R. Folman, and J. Schmiedmayer, Science 319, 1226 (2008).

${ }^{6}$ S. Kraft, A. Günther, H. Ott, D. Wharam, C. Zimmermann, and J. Fortágh, J. Phys. B: At. Mol. Opt. Phys. 35, L469 (2002).

7 A. E. Leanhardt, A. P. Chikkatur, D. Kielpinski, Y. Shin, T. L. Gustavson, W. Ketterle, and D. E. Pritchard, Phys. Rev. Lett. 89, 040401 (2002).
${ }^{8}$ M. P. A. Jones, C. J. Vale, D. Sahagun, B. V. Hall, and E. A. Hinds, Phys. Rev. Lett. 91, 080401 (2003).

9 P. Krüger et. al. , J. Phys.: Conf. Ser. 19, 56 (2005); Phys. Rev. A 76, 063621 (2007).

10 J. Estéve, C. Aussibal, T. Schumm, C. Figl, D. Mailly, I. Bouchoule, C. I. Westbrook, and A. Aspect, Phys. Rev. A 70, 043629 (2004).

11 D.-W. Wang, M. D. Lukin, and E. Demler, Phys. Rev. Lett. 92, 076802 (2004).

12 T. Schumm, J. Estéve, C. Figl, J.-B. Trebbia, C. Aussibal, H. Nguyen, D. Mailly, I. Bouchoule, C. I. Westbrook, and A. Aspect, Euro. Phys. J. D 32, 171 (2005).

13 R. Folman et. al., Adv. At. Mol. Opt. Phys. 48, 263 (2002); J. Fortagh and C. Zimmermann, Rev. Mod. Phys. 79, 235 (2007).

14 T. David, Y. Japha, V. Dikovsky, R. Salem, C. Henkel and R. Folman, in preparation.

15 Our treatment gives a full solution for small arbitrary fluc- 
tuations in a wire with rectangular cross section. The case of top surface fluctuations was given in Ref. 12 only for symmetric fluctuations that contribute to the magnetic field above the middle of the wire.

16 Y. Japha, O. Entin-Wohlman and R. Folman, unpublished. 